\title{
Knowledge, attitudes and prevention practices regarding HIV/AIDS among barbers in Ho municipality, Ghana
}

\author{
Mercy Quarm ${ }^{1}$, Elvis Tarkang ${ }^{2,3}$, Lilian Pencille ${ }^{2}$, Prosper Lutala ${ }^{4}$
}

\begin{abstract}
1. School of Public Health, University of Health and Allied Sciences PMB 31 Ho, Ghana, Department of Population and Behavioural Sciences.

2. HIV/AIDS Prevention Research Network Cameroon PO Box 36 Kumba, Cameroon (HIVPREC).

3. Department of Population and Behavioural Sciences, School of Public Health, University of Health and Allied Sciences PMB 31 Ho, Ghana.

4. College of Medicine, School of Public Health \& Family Medicine, Department of Family Medicine.
\end{abstract}

\begin{abstract}
Barbering poses risks of HIV transmission if it is not conducted in a hygienic manner. This study assessed the knowledge, attitudes and prevention practices regarding HIV transmission among barbers in the Ho Municipality, Ghana. A cross-sectional design was employed in Ho Municipality, and data were collected from 121 participants and analysed using Stata version 13.0 at the significance level of 0.05 . Knowledge was low $(63.6 \%)$ and was associated with attitude [COR=4.37 (95\% CI: 1.98-9.62); $\mathrm{p}<0.001]$ and with level of education [COR $=4.92(95 \% \mathrm{CI}: 1.70-14.21) ; \mathrm{p}=0.003]$. Attitude was inadequate $(58.7 \%)$ and was associated with level of education [COR=3.47 (95\% CI: 1.18-10.14); $\mathrm{p}=0.023$ ]. Poor prevention practice was high (87.6\%) and was associated with work experience [COR=20.72 (95\% CI: 3.86-111.05); $\mathrm{p}<0.001$ ] and location of operation [COR=4.92 (95\% CI: 1.60-15.14); $\mathrm{p}=0.006$. Programmes aimed at improving knowledge, attitudes and practices should focus on Barbers in urban communities, those without any fprmal education, and those with less than five years work experience.

Keywords: Knowledge, attitudes and practice, HIV/AIDS, Barbers, Ghana.

DOI: https://dx.doi.org/10.4314/ahs.v20i3.9

Cite as: Quarm M, Tarkang E, Pencille L, Lutala P. Knowledge, attisumestand prevention practices regarding HIV / AIDS among barbers in

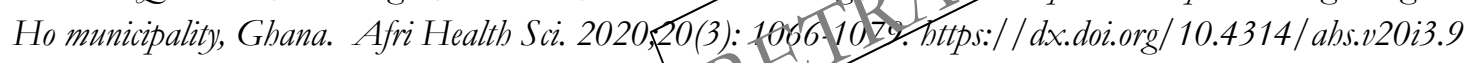

\section{Background}

HIV/AIDS remains a major public health problem all over the world, particularly in sub-Saharan Africa (SSA) where it has caused incalculable human suffering, social and cultural disruption and huge economic losses ${ }^{1}$. United Nations AIDS (UNAIDS) estimates 36.7 million people are living with HIV (PLHIV) globally, and among them 25.6 million livein SSA(1). UNAIDS estimates the number of PLHIV in Ghana at 274,600, representing a prevalence of $2.4 \%$ and accounting for 9,200 deaths annually ${ }^{1}$.

\section{Corresponding author:}

Prosper Lutala,

College of Medicine University of Malawi,

Family medicine

Email: plutala@medcol.mw, jolutprosper@yahoo.fr
HIV can be transmitted through unsafe use of therapeutic injections, blood transfusions, mother-to-child transmission (MTCT), unsafe sexual practices, and some beauty treatments like tattooing, piercing, pedicure and barbershop shaving with unsterilized instruments $^{2}$.

Given the predominance of several routes of transmission over others ${ }^{1}$, efforts so far have focused mainly on prevention of HIV through sex, blood transfusion and MTCT.

HIV transmission through sharing of non-sterile sharp instruments such as those used for barbing, circumcision, facial scarification, incision, tattooing, ear perforation, bloodletting, injections and acupuncture have always been vaguely classified as 'others' and given less attention in the campaign against the spread of HIV.

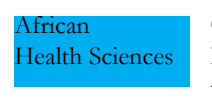

(C) 2020 Quarm M et al. Licensee African Health Sciences. This is an Open Access article distributed under the terms of the Creative commons Attribution License (https://creativecommons.org/licenses/BY/4.0), which permits unrestricted use, distribution, and reproduction in any medium, provided the original work is properly cited. 
Barbers are workers that undertake skin-piercing practices involving re-useable sharp instruments, which pose the risk of transmission of HIV and other blood-borne pathogens from one client to another. Though barbers do not carry out procedures that deliberately penetrate the skin, their procedures can inadvertently damage the skin through abrasion or minor accidental cuts ${ }^{3}$. These sharp equipment are not often sterilized, thus exposing clients to blood-borne pathogens, including HIV ${ }^{4}$.

A simple nick caused by a clipper or razor blade is enough for HIV infection to occur ${ }^{5}$. Due to the lipid envelope that protects HIV from dehydration, the virus can survive on the surfaces of barbing instruments for a period long enough for transmission to occur, particularly in commercial barber shops ${ }^{6}$.

Studying knowledge, attitudes and practices regarding HIV among barbers is of real interest as several studies have shown that contaminated tools useet by bariors are a source of transmission of $\mathrm{HIV}^{7-9}$. There is little research regarding knoul hode, attitudes and prevention practices of barbers in Ghana with respect to the transmission of $\mathrm{HIV}^{10}$. This study was therefore conducted to assess the knowledge, attitudes and prevention practices regarding HIV/AIDS among barbers in the Ho Municipality, Ghana.

\section{Materials and method}

\section{Study site description}

The Ho Municipality found in the Volta region of Ghana is located between latitudes $6^{\circ} 20^{\circ} " \mathrm{~N}$ and $6^{\circ} 55^{\circ} " \mathrm{~N}$ and longitudes $0^{\circ} 12^{\circ}$ " $\mathrm{E}$ and $0^{\circ} 53^{\circ}$ " $\mathrm{E}$. The municipality shares boundaries with Adaklu and Agortime-Ziope Districts to the South, Ho West District to the NorthWest and the Republic of Togo to the East. Its total land area is 2,361 square kilometers, representing 11.5 percent of the region's total land area. The population of Ho Municipality according to the 2010 Population and Housing Census is 177,281 , representing 8.4 percent of the region's total population. Females constitute 52.7 percent and males represent 47.3 percent. About 62 percent of the population resides in urban localities. The youth (those younger than 15 years) in the Municipality account for 31 percent of the population; there are a small number of elderly persons aged 65 years and older. The employed population represents 47.3 percent. Of the employed population about 21.4 percent are engaged as skilled agricultural, forestry and fishery workers, 26.8 percent are engaged in service and sales, 22.6 percent are involved in craft and related trade, and 15.8 percent are managers, professionals, and technicians $^{13}$.
Ho, the Capital of the Volta region of Ghana, has an HIV prevalence of $2.6 \%$, which is above the national prevalence $^{12}$.

\section{Study population}

All male Barbers in the Municipality who consented were considered in this study. Participants were sampled from the barbers' list taken from the Ghana National Association of Barbers, Ho Secretariat,using a simple random sampling technique.

\section{Inclusion and exclusion criteria}

Male Barbers working in barber shops who consented to be part of the study were included. Road-side barbers were excluded.

\section{D \\ Study design}

A cross-sectional descriptive design was used to determine the knowledge, attitudes and prevention practices of Barbers regarding HIV transmission in the Ho Municipality using structured questionnaires as the data collection tool. This design allows for quick and easy data gathering even from a large target population.

The snapshot nature of cross-sectional studies, while convenient, is limited in that it does not provide a good basis for establishing causality. Three distinct variables were measured in the current study at the same point in time, and thus it could not positively be determined if one caused the other. This uncertainty was countered in the study by decreasing bias in the data collection process, as the questionnaire measured each variable separately and data analysis was performed on the various variables to ascertain if there were associations between them.

\section{Sample size determination}

The minimum sample size was obtained for this study by using Cochran formula, $\mathrm{n}=$, (Where $\mathrm{n}=$ Sample size, $\mathrm{Z}=\mathrm{Z}$ - score, $\mathrm{p}=$ estimated prevalence of an attribute that is present in the population, $q=1-p, d=$ margin of error

$$
\begin{aligned}
& n=\text { sample size } \\
& Z=Z \text {-score } \\
& P=\text { prevalence } \\
& q=1 \text {-prevalence } \\
& d=\text { Margin of error }
\end{aligned}
$$$$
\mathrm{n}=\text { ? }
$$$$
\mathrm{z}=95 \%=1.96
$$$$
\mathrm{p}=0.082
$$$$
\mathrm{q}=1-0.082
$$$$
\mathrm{d}=0.05
$$

Therefore, the minimum sample size for the current study was 121 . 


\section{Sampling method}

The Cluster sampling method was used to divide the municipality into clusters. A simple random sampling technique was used to sample the barbers from the barbers' list of the Ghana National Association of Barbers, Ho Secretariat. A cluster represented a sub-municipality within the Ho Municipality. The names of the communities in each cluster were written on pieces of paper and folded and shaken to ensure they mixed well. Using the lottery method, three communities were selected randomly from each sub-municipality. Barbers were then selected randomly from each selected community using the sampling frame of barbers provided by the Ho barbers' Association, until the required sample size was reached.

\section{Data collection procedure}

Data were collected using pretested struetured fues tionnaires. A sample of ten (10) questionndires tras pretested in some barbing salons in HoM m fricipality, which were not included in the actualstudy. The researcher used the barbers' responses during the pretesting to assess the clarity and suitability of the items in the questionnaire to the participants. Data collectors were trained to carefully collect the data. Barbers who were not willing to participate in the study were excluded.

\section{Data analysis}

Data were entered using Epi Data Software Version 3 and analyzed using Stata Version 13. Data were represented using tables and graphs; Chi-square and Logistic regression analyses were conducted at the level of significance of 0.05. Knowledge was assessed using 7 items and was rated as good if a barber had 5 or more correct answers and poor if a barber had fewer than 5 correct answers. Attitude was assessed using 4 items and was dichotomized into good attitude and poor attitude. Anyone who responded 'yes' to 3 or more questions was considered as having good attitude. On the other hand, anyone who answered 'yes' to fewer than 3 questions, was considered as having poor attitude towards HIV/
AIDS. Practices were assessed by rating them into good and bad practices. Anyone who answered more than 7 questions correctly was rated good, and those who answered fewer than 7 questions correctly were rated poor.

\section{Ethical issues}

Ethical approval for the study was sought from the Ghana Health Service Ethics Review Committee through the University of Health and Allied Sciences.

Permission was sought from the Ho Municipal Health Directorate and the Ghana National Association of barbers (GNAB) before the study was conducted. Participants were also assured that under no condition whatsoever would their names or any other contacts be linked io theldata analysis and dissemination of the findinfos to the study. It was made clear to the participants that all their responses would be confidential during and after the data collection. Furthermore, participants were assured that storage, analysis and reporting of all data including dissemination would be done in codes, hence identity of the respondents will not be exposed. In addition, an Informed Consent sheet, which provided details and willingness to participate in the study, was administered to the participants and they were required to designate their acceptance and approval to participate in the study.

\section{Results \\ Demographic and occupational characteristics}

Table 1 shows the demographic and occupational characteristics of the participants. The mean age of the participants was $26.5 \pm 5.63$. A majority of the participants, $81(66.9 \%)$ were aged $20-29$ years and a majority, $66(54.6 \%)$ were single. A majority, 54(44.6\%) had secondary education and most, 96(79.3\%) were Christians. A majority, 59(48.8\%) had less than 5 years work experience and most, 113(93.4\%) worked with fewer than 5 assistants. A majority, 53(43.8\%) attended to 1019 clients per day and most were located in the uran area, $93(76.9 \%)$.Most, $70(57.9 \%)$ learnt the profession through apprenticeship. 
Table 1: Demographics and occupational characteristics of Participants

\begin{tabular}{|c|c|c|}
\hline Variable & Frequency (N) & Percent (\%) \\
\hline Mean age (S.D) & $26.5( \pm 5.63)$ & \\
\hline \multicolumn{3}{|l|}{ Age group (Years) } \\
\hline$<20$ & 8 & 6.6 \\
\hline $20-29$ & 81 & 66.9 \\
\hline $30-39$ & 28 & 23.1 \\
\hline $40+$ & 4 & 3.4 \\
\hline \multicolumn{3}{|l|}{ Marital Status } \\
\hline Single & 66 & 54.6 \\
\hline Married & 39 & 32.2 \\
\hline Cohabiting & 13 & 10.7 \\
\hline Divorced & 3 & 2.5 \\
\hline \multicolumn{3}{|l|}{ Educational Level } \\
\hline None & 21 & 17.4 \\
\hline Primary & 42 & 34.7 \\
\hline Secondary & 54 & 44.6 \\
\hline Post-secondary & 4 & 3.3 \\
\hline \multicolumn{3}{|l|}{ Religion } \\
\hline Christian & & 79.3 \\
\hline Muslim & & 16.6 \\
\hline Traditional & & 4.1 \\
\hline \multicolumn{3}{|l|}{ Work Experience } \\
\hline$<5$ years & 59 & 48.8 \\
\hline $5-10$ years & 43 & 35.5 \\
\hline$>10$ years & 19 & 15.7 \\
\hline \multicolumn{3}{|c|}{ Number of Assistants per barber } \\
\hline$<5$ Assistants & 113 & 93.4 \\
\hline$>5$ Assistants & 8 & 6.6 \\
\hline \multicolumn{3}{|c|}{ Number of client attend per day } \\
\hline$<10$ & 45 & 37.2 \\
\hline $10-19$ & 53 & 43.8 \\
\hline $20+$ & 23 & 19.0 \\
\hline \multicolumn{3}{|l|}{ Location } \\
\hline Urban & 93 & 76.9 \\
\hline Rural & 28 & 23.1 \\
\hline \multicolumn{3}{|l|}{ Mode of learning } \\
\hline Apprenticeship & 70 & 57.9 \\
\hline Barbering school & 6 & 5.0 \\
\hline On the job training & 31 & 25.6 \\
\hline
\end{tabular}

\section{Knowledge of HIV/AIDS}

Table 2 describes the knowledge of HIV/AIDS on the part of the barbers. A majority, 114(94.2\%) had heard of HIV/AIDS and less than half, 58(47.9\%) knew HIV/AIDS was caused by a virus.
A majority, 88(72.7\%) knew barbers were at risk of infecting clients, and a majority,80(66.1\%) also knew clients were at risk of infecting barbers. A majority, $76(62.8 \%)$ knew HIV is preventable, and a majority, $82(67.8 \%)$ also knew sterilization of barbing instruments could prevent HIV transmission. 
Table 2: Knowledge on HIV/AIDS of Participants

\begin{tabular}{|c|c|c|}
\hline Variable & Frequency $(\mathbf{N})$ & Percent (\%) \\
\hline \multicolumn{3}{|c|}{ Heard of HIV/AIDS } \\
\hline Yes & 114 & 94.2 \\
\hline No & 7 & 5.8 \\
\hline \multicolumn{3}{|c|}{ Causes of HIV } \\
\hline Germs & 37 & 30.6 \\
\hline Virus & 58 & 47.9 \\
\hline Witchcraft & 14 & 11.7 \\
\hline Bacteria & 6 & 4.9 \\
\hline Others & 6 & 4.9 \\
\hline \multicolumn{3}{|c|}{ Barber's risk of infecting clients } \\
\hline Yes & 88 & 72.7 \\
\hline No & 14 & 11.6 \\
\hline Do not know & & 15.7 \\
\hline \multicolumn{3}{|c|}{ Client's risk of infecting barbers } \\
\hline Yes & & 66.1 \\
\hline No & 22 & 18.2 \\
\hline Do not know & 19 & 15.7 \\
\hline \multicolumn{3}{|c|}{ Is HIV preventable? } \\
\hline Yes & 76 & 62.8 \\
\hline No & 22 & 15.8 \\
\hline Do not know & 19 & 21.4 \\
\hline \multicolumn{3}{|c|}{ Does sterilization prevent HIV? } \\
\hline Yes & 82 & 67.8 \\
\hline No & 7 & 5.7 \\
\hline Do not know & 32 & 26.5 \\
\hline
\end{tabular}

Knowledge level regarding HIV/AIDS

Figure 1 depicts the overall level of knowledge of bar- bers regarding HIV/AIDS. Of the 121 participants, most, 77(63.6\%) had poor knowledge of HIV/AIDS.

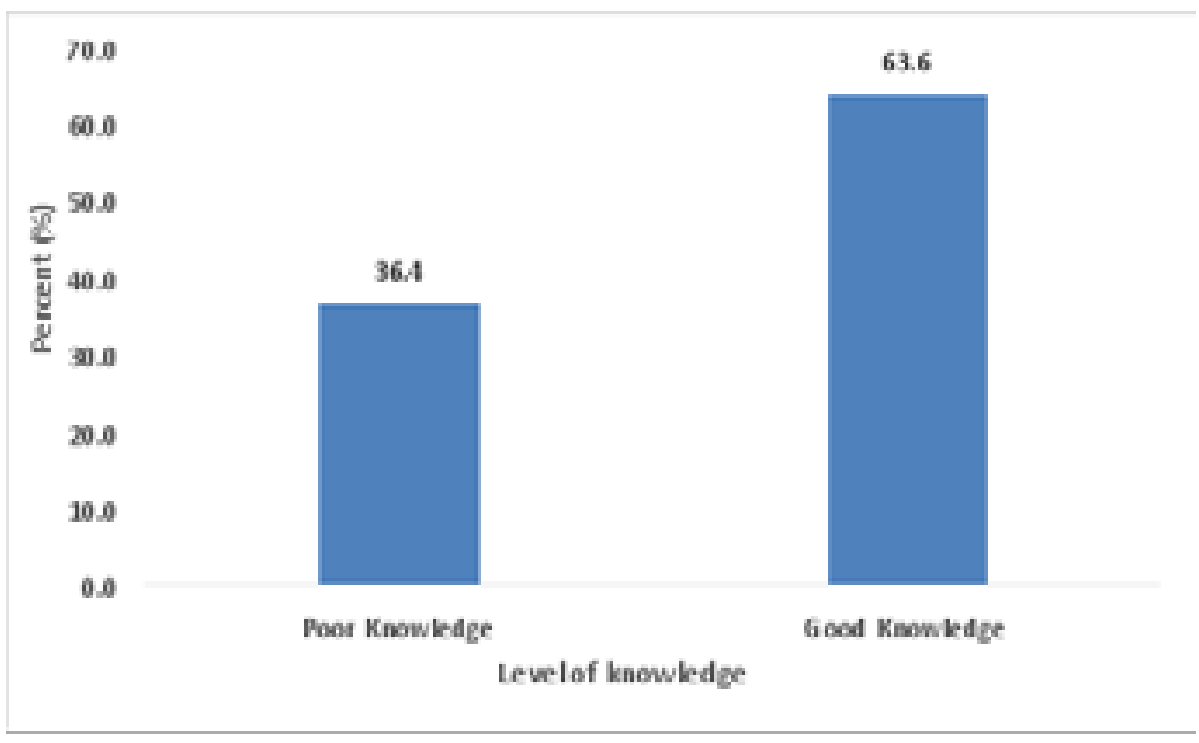

Figure 1: Overall level of knowledge of Barbers on HIV/AIDS 
Association between knowledge regarding HIV/ AIDS and demographic characteristics

Table 3 shows the association between participants' knowledge regarding HIV/AIDS and demographic characteristics. Barbers with primary and secondary education were more likely to have good knowledge regarding HIV/AIDS than those with no format education $[\mathrm{COR}=3.43(95 \% \mathrm{CI}: 1.16-10.09) ; \mathrm{p}=0.025 \mathrm{~A}$ a d [COR=4.92 (95\% CI: 1.70-14.21); p =0.003], Cesbectitely. Barbers with 5-10 years' work experterce nere more likely to have good knowledge regareting HIV/AIDS than those with $<5$ years' work experience $[\mathrm{COR}=2.78$ (95\% CI: 1.16-6.66); $\mathrm{p}=0.002]$. Barbers with good attitudes towards HIV/AIDS were more likely to have good knowledge regarding HIV/AIDS [COR $=4.37$ (95\% CI: 1.98-9.62); $\mathrm{p}<0.001]$. However, barbers who were cohabiting were less likely to have good knowld dige pegarding HIV/AIDS than those who were single YOR $=0.20$ (95\% CI: 0.06-0.71); $\mathrm{p}=0.013$ ]. In the same vein, barbers who had 10-19 clients a day were less likely to have good knowledge regarding HIV/AIDS than those who had $<10$ clients a day $[\mathrm{COR}=0.34(95 \% \mathrm{CI}$ : 0.14-0.83); $\mathrm{p}=0.019]$.

Table 3: Association between demographic characteristics of barbers and odds of knowledge

\begin{tabular}{|c|c|c|c|c|c|}
\hline Variable & \multicolumn{2}{|c|}{ Knowledge } & \multirow{2}{*}{$\begin{array}{l}\text { Chi-square } \\
\mathbf{X}^{2} \text { (p-value) }\end{array}$} & \multirow[t]{2}{*}{ COR(95\%CI)p-value } & \multirow[t]{2}{*}{ AOR(95\%CI)p-value } \\
\hline Age group & $\begin{array}{c}\text { Good } \\
\text { knowledge } \\
{[\mathrm{N}=77]}\end{array}$ & $\begin{array}{c}\text { Poor knowledge } \\
{[\quad[N=44]}\end{array}$ & & & \\
\hline$<20$ & $5(6.5)$ & $3(6.8)$ & & & \\
\hline $20-29$ & $50(64.9)$ & $31(70.5)$ & & $0.96(0.21,4.33) 0.966$ & $0.97(0.13,7.07) 0.983$ \\
\hline $30-39$ & $21(27.3)$ & $7(15.9)$ & & $1.8(0.33,9.53) 0.490$ & $0.84(0.06,10.76) 0.896$ \\
\hline $40+$ & $1(1.3)$ & $3(6.8)$ & $4.27(0.233)$ & $0.2(0.01,2.91) 0.239$ & $0.18(0.00,8.77) 0.393$ \\
\hline \multicolumn{6}{|l|}{ Marital Status } \\
\hline Single & $46(59.7)$ & $20(45.5)$ & & & \\
\hline Married & $24(31.2)$ & $15(34.1)$ & & $0.69(0.30,1.58) 0.389$ & $0.33(0.07,1.54) 0.161$ \\
\hline Cohabiting & $4(5.2)$ & $9(20.4)$ & & $0.20(0.06,0.71) 0.013$ & $0.07(0.01,0.54) 0.010$ \\
\hline Divorced & $3(3.9)$ & $0(0.0)$ & $8.90(0.031)$ & $3.08(0.15,62.50) 0.463$ & $1.03(0.01,88.80) 0.987$ \\
\hline \multicolumn{6}{|l|}{ Educational Level } \\
\hline None & $7(9.1)$ & $14(31.8)$ & & & \\
\hline Primary & $27(35.1)$ & $15(34.1)$ & & $3.43(1.16,10.09) 0.025$ & $4.34(0.91,20.69) 0.065$ \\
\hline Secondary & $39(50.7)$ & $15(34.1)$ & & $4.92(1.70,14.21) 0.003$ & $8.74(1.95,39.11) 0.005$ \\
\hline Post-secondary & $4(5.1)$ & $0(0.0)$ & $12.34(0.006)$ & $17.40(0.82,368.08) 0.067$ & $41.19(1.15,1471.84) 0.042$ \\
\hline \multicolumn{6}{|c|}{ 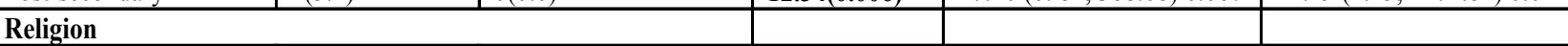 } \\
\hline Christian & $63(81.8)$ & $33(75.0)$ & & & \\
\hline Muslim & $13(16.9)$ & $7(15.9)$ & & $0.97(0.35,2.67) 0.957$ & $0.90(0.25,3.27) 0.883$ \\
\hline Traditional & $1(1.3)$ & $4(9.1)$ & $4.29(0.117)$ & $0.13(0.01,1.22) 0.074$ & $0.38(0.03,4.17) 0.434$ \\
\hline \multicolumn{6}{|l|}{ Work Experience } \\
\hline$<5$ years & $32(41.5)$ & $27(61.4)$ & & & \\
\hline 5-10 years & $33(42.9)$ & $10(22.7)$ & & $2.78(1.16,6.66) 0.022$ & $13.84(2.67,71.63) 0.002$ \\
\hline$>10$ years & $12(15.6)$ & $7(15.9)$ & $5.44(0.066)$ & $1.44(0.49,4.18) 0.496$ & $8.42(0.64,109.7) 0.104$ \\
\hline \multicolumn{6}{|c|}{ Number of apprentice per barber } \\
\hline$<5$ Assistants & $73(94.8)$ & $40(90.9)$ & & & \\
\hline$>5$ Assistants & $4(5.2)$ & $4(9.1)$ & $0.68(0.407)$ & $0.54(0.13,2.30) 0.412$ & $0.14(0.00,2.15) 0.159$ \\
\hline \multicolumn{6}{|c|}{ Number of client attendance per day } \\
\hline$<10$ & $35(45.5)$ & $10(22.7)$ & & & \\
\hline $10-19$ & $29(37.6)$ & $24(54.6)$ & & $0.34(0.14,0.83) 0.019$ & $0.14(0.03,0.57) 0.006$ \\
\hline $20+$ & $13(16.9)$ & $10(22.7)$ & $6.21(0.045)$ & $0.37(0.12,1.09) 0.073$ & $0.14(0.04,1.34) 0.107$ \\
\hline \multicolumn{6}{|l|}{ Location } \\
\hline Urban & $60(77.9)$ & $33(75.0)$ & & & \\
\hline Rural & $17(22.1)$ & $11(25.0)$ & $0.13(0.714)$ & $0.85(0.35,2.02) 0.714$ & $0.26(0.06,1.11) 0.070$ \\
\hline \multicolumn{6}{|l|}{ Mode of operation } \\
\hline Apprenticeship & $45(58.4)$ & $25(56.8)$ & & & \\
\hline Barbering school & $5(6.5)$ & $1(2.3)$ & & $2.77(0.30,25.11) 0.363$ & $3.67(0.05,237.0) 0.540$ \\
\hline On the job training & $15(19.5)$ & $16(36.4)$ & & $0.52(0.22,1.22) 0.136$ & $0.47(0.13,1.67) 0.247$ \\
\hline Others & $12(15.6)$ & $2(4.5)$ & $7.08(0.069)$ & $3.33(0.69,16.09) 0.134$ & $7.21(0.56,91.77) 0.128$ \\
\hline \multicolumn{6}{|l|}{ Attitude } \\
\hline Poor attitude & $28(63.6)$ & $22(28.6)$ & & & \\
\hline Good attitude & $16(36.4)$ & $55(71.4)$ & $14.19(<0.001)$ & $4.37(1.98,9.62) 0.000$ & $3.64(1.11,11.92) 0.032$ \\
\hline
\end{tabular}


Attitude of barbers regarding HIV/AIDS

Table 4 shows the attitudes of barbers regarding HIV/AIDS. A majority, 87(71.9\%) had not tested for HIV/AIDS before. Of the 121 participants, a majority, 83(68.6\%) agreed that they would attend to clients who are HIV-positive. The majority of the participants,
$104(85.9 \%)$ agreed that they would continue with their profession if they became HIV-positive. The majority, $50(41.3 \%)$ were willing to attend to a client who disclosed their HIV status to them, while 14(11.6\%) would have attended, but sterilized the instruments after use. Overall, majority, $71(58.7 \%)$ portrayed a good attitude regarding HIV/AIDS.

Table 4: Attitude of Barbers regarding HIV/AIDS

\begin{tabular}{|c|c|c|}
\hline Variables & $\begin{array}{l}\text { Number } \\
\mathrm{N}=[121]\end{array}$ & $\begin{array}{l}\text { Percentage } \\
(\%)\end{array}$ \\
\hline \multicolumn{3}{|l|}{ Have you test for HIV/AIDS before? } \\
\hline Yes & 34 & 28.1 \\
\hline No & 87 & 71.9 \\
\hline \multicolumn{3}{|l|}{ Will you attend to client with HIV? } \\
\hline \multicolumn{3}{|l|}{ Yes } \\
\hline \multicolumn{3}{|l|}{ No } \\
\hline \multicolumn{3}{|c|}{ Will you continue your profession if you have AIDS? } \\
\hline Yes & & 85.9 \\
\hline No & 17 & 14.1 \\
\hline \multicolumn{3}{|c|}{ How will you attend to client with who discloses his $x$. Sattus to you? } \\
\hline Welcome the person & 50 & 41.3 \\
\hline Reject the person & 23 & 19.0 \\
\hline Welcome, but will sterilize afterwards & 14 & 11.6 \\
\hline Others & 34 & 28.1 \\
\hline \multicolumn{3}{|c|}{ Overall Attitude towards HIV/AIDS Clients } \\
\hline Good Attitude & 71 & 58.7 \\
\hline Bad Attitude & 50 & 41.3 \\
\hline
\end{tabular}

Association between demographic characteristic and attitude regarding HIV/AIDS

Table 5 shows the association between demographic characteristics and attitude regarding HIV/AIDS. Barbers with primary education were more likely to have good attitudes towards HIV/AIDS than those with no formal education $[\mathrm{COR}=3.47$ (95\% CI: 1.18-10.14); $\mathrm{p}=0.023]$.

In the same vein, barbers with more than 10 years' work experience were more likely to have good attitudes to- wards HIV/AIDS than those with less than 5 years' work experience $[\mathrm{COR}=4.5$ (95\% CI: 1.18-17.10); $\mathrm{p}=0.027$. Likewise, barbers with good knowledge regarding HIV/AIDS were more likely to have good attitudes regarding HIV/AIDS than those with poor knowledge [COR=4.37 (95\% CI: 1.98-9.62) p <0.001]. However, barbers who had on-job training were less likely to have good attitudes regarding HIV/AIDS than those who were trained through apprenticeship [COR=0.26 (95\% CI: 0.11-0.65); $\mathrm{p}=0.004]$. 
Table 5: Association between demographic characteristics of Barbers and odds of Attitude

\begin{tabular}{|c|c|c|c|c|c|}
\hline Variable & \multicolumn{2}{|c|}{ Attitude } & \multirow{2}{*}{$\begin{array}{l}\text { Chi-square } \\
\mathrm{X}^{2} \text { (p-value) }\end{array}$} & \multirow[t]{2}{*}{ COR(95\%CI)p-value } & \multirow[t]{2}{*}{ A0R(95\%CI)p-value } \\
\hline $\begin{array}{l}\text { Educational } \\
\text { Level }\end{array}$ & $\begin{array}{c}\text { Poor } \\
\text { Attitude } \\
{[\mathrm{N}=50]}\end{array}$ & $\begin{array}{l}\text { Good Attitude } \\
\qquad[\mathrm{N}=71]\end{array}$ & & & \\
\hline $\begin{array}{l}\text { No } \\
\text { formal education }\end{array}$ & $13(26.0)$ & $8(11.3)$ & & & \\
\hline Primary & $13(26.0)$ & $29(40.8)$ & & $3.47(1.18,10.14) 0.023$ & $4.02(0.84,19.07) 0.079$ \\
\hline Secondary & $24(48.0)$ & $30(42.3)$ & & $1.97(0.72,5.42) 0.186$ & $2.71(0.55,13.19) 0.216$ \\
\hline Post-secondary & $0(0.0)$ & $4(5.6)$ & $8.56(0.036)$ & $14.29(0.68,300.37) 0.087$ & $21.97(0.56,854.8) 0.098$ \\
\hline \multicolumn{6}{|l|}{ Religion } \\
\hline Christian & $38(76.0)$ & $58(81.7)$ & & & \\
\hline Muslim & $10(20.0)$ & $10(14.1)$ & & $0.65(0.24,1.72) 0.392$ & $1.08(0.33,3.50) 0.898$ \\
\hline Traditional & $2(4.0)$ & $3(4.2)$ & $0.74(0.689)$ & $0.98(0.15,6.15) 0.985$ & $1.08(0.03,29.83) 0.961$ \\
\hline \multicolumn{6}{|l|}{ Work Experience } \\
\hline$<5$ years & $27(54.0)$ & $32(45.1)$ & & & \\
\hline $5-10$ years & $20(40.0)$ & $23(32.4)$ & & $0.97(0.44,2.13) 0.940$ & $0.96(0.27,3.36) 0.959$ \\
\hline$>10$ years & $3(6.0)$ & $16(22.5)$ & $5.44(0.066)$ & $4.5(1.18,17.10) 0.027$ & $2.65(0.26,26.26) 0.404$ \\
\hline \multicolumn{6}{|l|}{ Mode of learning } \\
\hline Apprenticeship & $23(46.0)$ & $47(66.2)$ & & & \\
\hline Barbering school & $4(8.0)$ & $2(2.8)$ & & $0.24(0.04,1.43) 0.119$ & $0.23(0.03,1.56) 0.134$ \\
\hline $\begin{array}{l}\text { On the job } \\
\text { training }\end{array}$ & $20(40.0)$ & $11(15.5)$ & & $0.26(0.11,0.65) 0.004$ & $0.21(0.06,0.72) 0.013$ \\
\hline Others & $3(6.0)$ & $11(15.5)$ & $12.82(0.005)$ & $1.7(0.45,7.06) 0.403$ & $0.63(0.11,3.55) 0.606$ \\
\hline \multicolumn{6}{|l|}{$\begin{array}{l}\text { Community of } \\
\text { operation }\end{array}$} \\
\hline Urban & $42(84.0)$ & $51(71.5)$ & & & \\
\hline Rural & $8(16.0)$ & $20(28.5)$ & $2.44(0.118)$ & $1.53(0.40,5.14) 0.531$ & $2.05(0.82,5.84) 0.122$ \\
\hline \multicolumn{6}{|l|}{ Knowledge Level } \\
\hline Poor Knowledge & $28(56.0)$ & $16(22.5)$ & & & \\
\hline Good Knowledge & $22(44.0)$ & $55(77.5)$ & $14.19(<0.001)$ & $4.37(1.98,9.62)<0.001$ & $3.96(1.20,13.03) 0.023$ \\
\hline \multicolumn{6}{|l|}{ practices } \\
\hline Poor Practices & $47(94.0)$ & $59(83.10)$ & & & \\
\hline Good Practices & $3(6.0)$ & $12(16.9)$ & $3.21(0.073)$ & $3.18(0.34,11.95) 0.086$ & $1.55(0.21,11.20) 0.664$ \\
\hline
\end{tabular}

Prevention practices of barbers fegarding Hiv A majority, 104(85.9\%) cleaned their instruments imAIDS mediately after use on a client, and most, 45(37.2\%) Table 6 shows the prevention practices of barbers regarding HIV/AIDS. Among the 121 participants, the majority, 101(83.5\%) used razors and clippers on clients. Similarly, majority, 61(50.4\%) demonstrated hand washing in between clients, and the majority, 76(62.8\%) used water and disinfectants to clean their instruments. demonstrated disinfection and hand washing after every client.

Of the 121 participants, a majority, 75(62.0\%) had UV lights; majority $61(50.4 \%$ ) could not remember how frequently they had changed their UV lights. Overall, the majority, 106(87.6\%) exhibited poor HIV prevention practices. 
Table 6: Prevention practices of Barbers regarding HIV/AIDS

\begin{tabular}{l} 
Variables \\
\hline Do you use razors and clippers on clients? \\
Yes \\
No
\end{tabular}

Among the 121 participants, majority, 62.8\% used clippers.

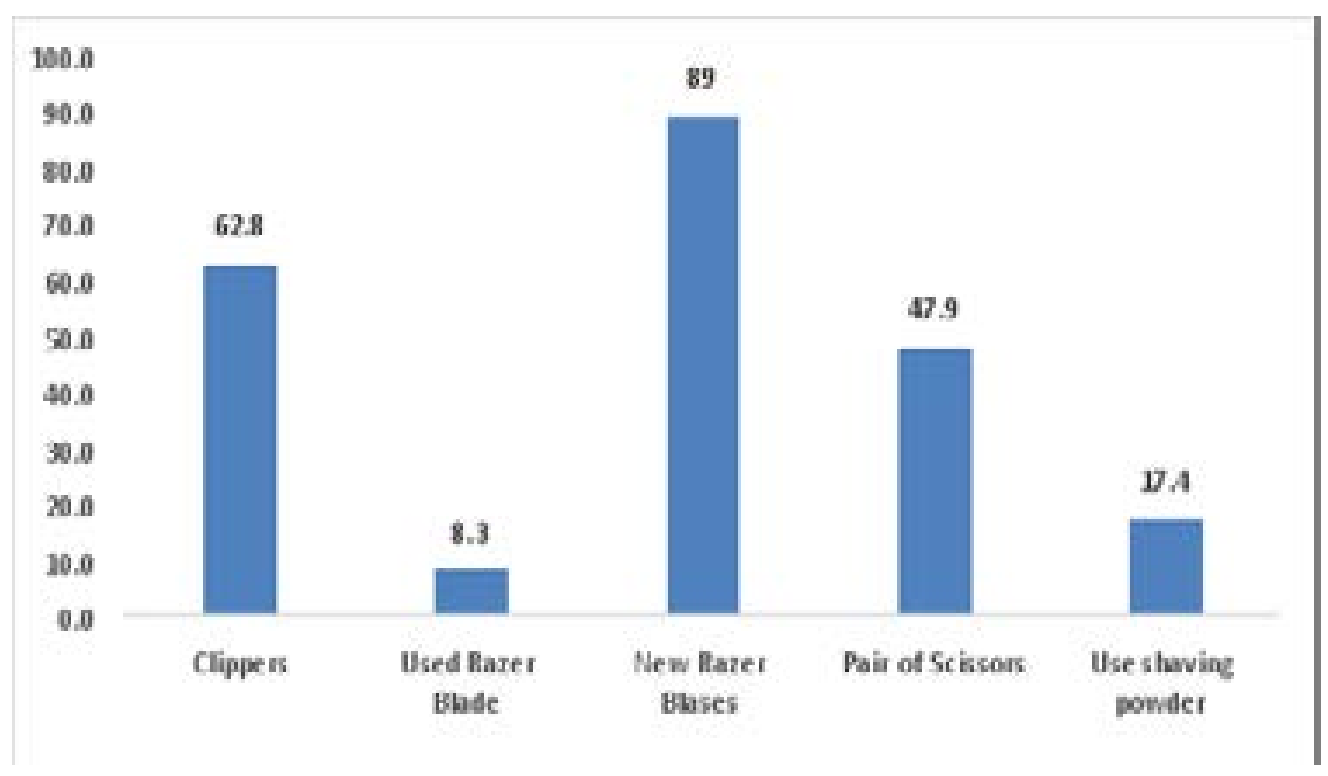

Figure 2: Instruments used for barbing hair 
As demonstrated in Figure 3,among the participants amajority, 58.7\% cleaned with soap and water.

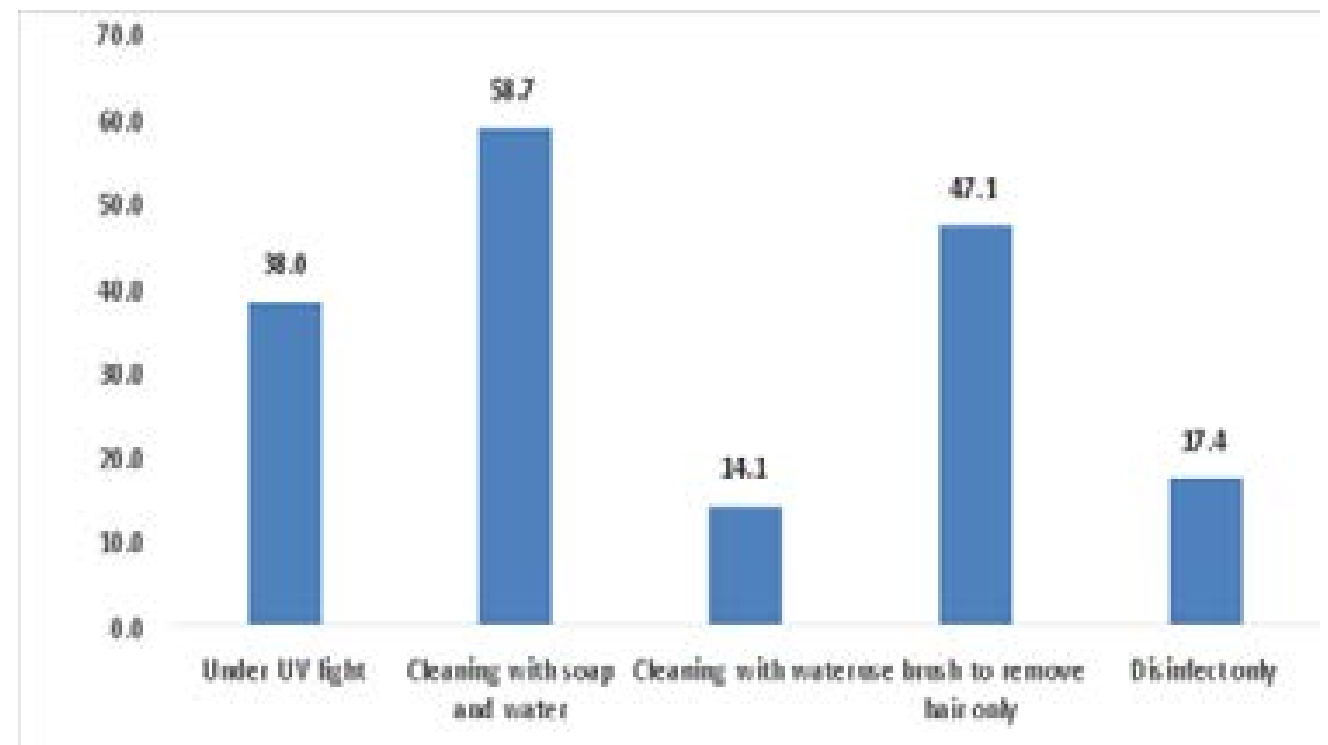

Figure 3: Methods used for sterilization barbing instruments

Among the 121 participants in the study, majority, $72.7 \%$ us d ndthylated spirit as disinfectant (see Figure

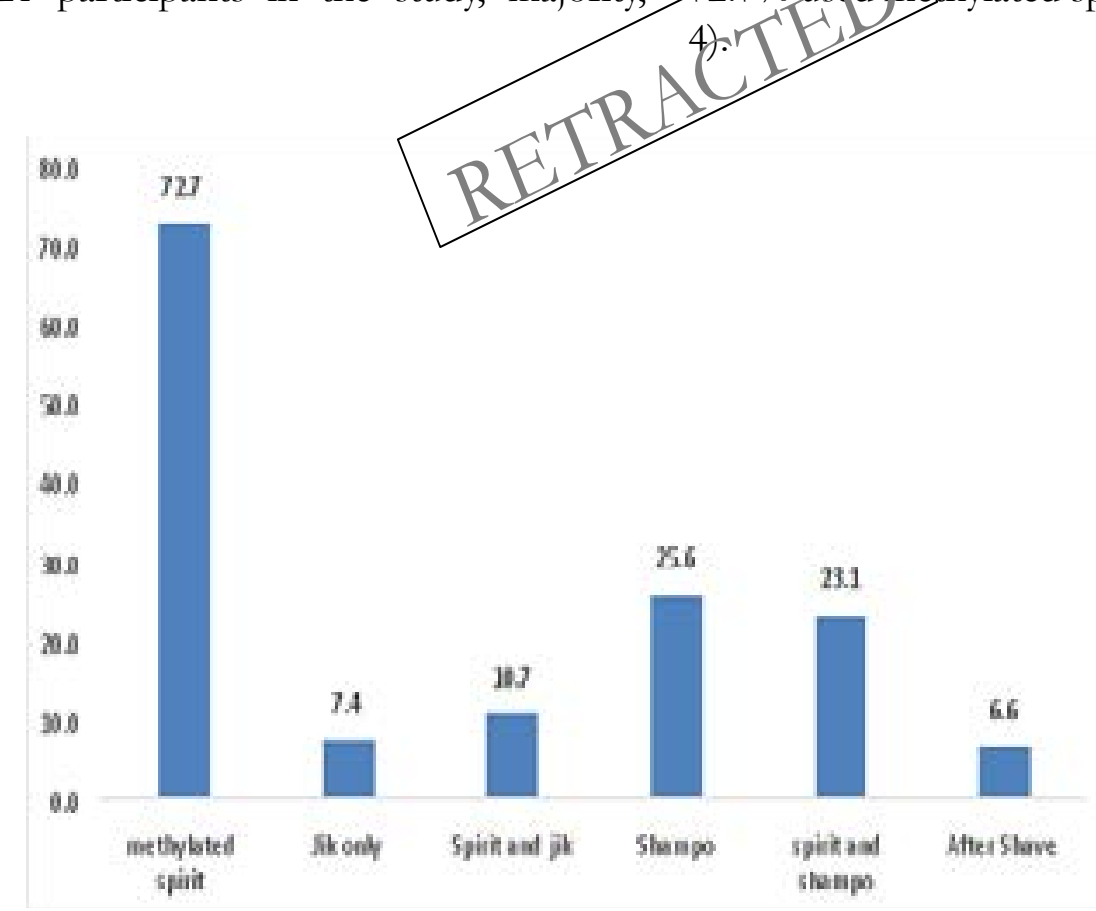

Figure 4: Disinfectants commonly used

Association between prevention practices and demographic characteristics

Table 7 shows the association between practices and demographic characteristics. Barbers who had $>10$ years' work experience were more likely to exhibit good HIV prevention practices than those with $<5$ years' work experience $[\mathrm{COR}=20.72$ (95\% CI: 3.86-111.05); $\mathrm{p}<0.001]$. In the same vein, barbers who were operating in rural communities were more likely to exhibit good HIV prevention practices than those who were operating in urban communities [COR $=4.92$ (95\% CI: 1.60 15.14); $\mathrm{p}=0.006$. 
Table 7: Association between demographic characteristics of Barbers and odds of practices

\begin{tabular}{|c|c|c|c|c|c|}
\hline \multirow{2}{*}{$\begin{array}{l}\text { Variable } \\
\text { Age group }\end{array}$} & \multicolumn{2}{|c|}{ Practices } & \multirow{2}{*}{$\begin{array}{l}\text { Chi-square } \\
\mathrm{X}^{2} \text { (p-value) }\end{array}$} & \multirow[t]{2}{*}{ COR(95\%CI)p-value } & \multirow[t]{2}{*}{ A0R(95\%CI)p-value } \\
\hline & $\begin{array}{c}\text { Poor } \\
\text { practices } \\
{[\mathrm{N}=106]}\end{array}$ & $\begin{array}{c}\text { Good practices } \\
{[N=15]}\end{array}$ & & & \\
\hline$<20$ & $8(7.6)$ & $0(0.0)$ & & & \\
\hline $20-29$ & $73(68.9)$ & $8(53.3)$ & & $1.96(0.10,37.15) 0.652$ & $1.51(0.07,33.90) 0.796$ \\
\hline $30-39$ & $22(20.7)$ & $6(40.0)$ & & $4.91(0.24,96.95) 0.296$ & $0.56(0.01,23.71) 0.759$ \\
\hline $40+$ & $3(2.8)$ & $1(6.7)$ & $4.29(0.231)$ & $7.28(0.23,225.89) 0.257$ & $0.35(0.00,50.98) 0.681$ \\
\hline \multicolumn{6}{|l|}{ Educational level } \\
\hline No formal education & $19(17.9)$ & $2(13.3)$ & & & \\
\hline Primary & $36(33.9)$ & $6(40.0)$ & & $1.38(0.29,6.60) 0.679$ & $1.16(0.13,10.44) 0.895$ \\
\hline Secondary & $47(44.4)$ & $7(46.7)$ & & $1.23(0.26,5.66) 0.789$ & $1.49(0.18,12.42) 0.711$ \\
\hline Post-secondary & $4(3.8)$ & $0(0.0)$ & $0.87(0.830)$ & $0.86(0.33,21.36) 0.930$ & $1.10(0.02,63.18) 0.961$ \\
\hline \multicolumn{6}{|l|}{ Work Experience } \\
\hline$<5$ years & $57(53.7)$ & $2(13.3)$ & & & \\
\hline $5-10$ years & $38(35.9)$ & $5(33.3)$ & & $3.75(0.69,20.33) 0.930$ & $1.74(0.29,10.43) 0.543$ \\
\hline$>10$ years & $11(10.4)$ & $8(53.4)$ & $19.87(0.000)$ & $20.72(3.86,111.05)<0.001$ & $10.87(0.72,165.07) 0.085$ \\
\hline \multicolumn{6}{|l|}{ Mode of learning } \\
\hline Apprenticeship & $61(57.6)$ & $9(60.0)$ & & & \\
\hline Barbering school & $6(5.6)$ & $0(0.0)$ & & $0.50(0.03,9.58) 0.644$ & $0.80(0.03,21.15) 0.894$ \\
\hline On the job training & $29(27.4)$ & $2(13.3)$ & & $0.55(0.13,2.36) 0.420$ & $0.99(0.15,6.61) 0.995$ \\
\hline Others & $10(9.4)$ & $4(26.7)$ & $5.24(0.155)$ & $2.77(0.76,10.17) 0.124$ & $0.46(0.07,2.97) 0.412$ \\
\hline \multicolumn{6}{|l|}{$\begin{array}{l}\text { Community of } \\
\text { operation }\end{array}$} \\
\hline Urban & $86(81.3)$ & $7(46.7)$ & & & \\
\hline Rural & $20(18.7)$ & $8(53.3)$ & $8.77(0.003)$ & $4.91(1.60,15.14) 0.006$ & $3.17(0.72,13.92) 0.127$ \\
\hline \multicolumn{6}{|l|}{ Attitude } \\
\hline Poor & $47(44.3)$ & & & & \\
\hline Good & $59(55.7)$ & $12(80.0)$ & $3.21,(0.013)$ & $3.19(0.85,11.95) 0.086$ & $1.07(0.17,6.69) 0.940$ \\
\hline \multicolumn{6}{|l|}{ Knowledge } \\
\hline Poor & $42(39.6)$ & & & & \\
\hline Good & $64(60.4)$ & 35(86.7) & $3.92(0.048)$ & $4.27(0.92,19.87) 0.065$ & $6.56(0.94,45.63) 0.058$ \\
\hline
\end{tabular}

\section{Discussion}

This cross-sectional study examined the knowledge, attitudes and prevention practices among barbers in Ho, Ghana, regarding HIV/AIDS. HIV/AIDS infection has been widely investigated in many occupational groups, but few data are available on the intensity and severity of the disease in barber shops in Ghana as a whole, and the Volta Region specifically. This study was intended to fill this gap.

The transmission of HIV/AIDS is usually considered to take place through blood transfusions, sexual contacts, MTCT and use of HIV-infected instruments. However, while barbing is not considered a major route of spread of the disease, and though barbers do not carry out procedures that deliberately penetrate the skin, their procedures can inadvertently damage the skin through abrasion or minor accidental cuts ${ }^{15}$. Despite this, barbers have not been given the needed at- tention to curb the spread of the disease. In part due to the high unemployment rate in the country, barbing has become a means of livelihood for many in the Ho municipality; however, there is little or no training on the standard protocol for barbing.

This study showed that majority of the barbers (63.6\%) had overall good knowledge regarding HIV/AIDS and $58.7 \%$ had good attitude regarding HIV/AIDS. However, majority $(87.6 \%)$ displayed poor prevention practices regarding HIV/AIDS.

The level of knowledge of HIV/AIDS of $63.6 \%$ among barbers is not in agreement with the Ghana Demographic and Health Survey, which claims 100\% knowledge of HIV/AIDS ${ }^{16}$. This difference could reflect the sample size used, or the literacy rate and population size in the GDHS; the data were obtained from the sentinel sites only. The HIV prevalence in Ghana is 
consistently higher among at-risk groups such as commercial sex workers, clients at STI clinics and long-distance truck drivers and as such the awareness, education and prevention campaigns are geared towards these groups. These sub-populations with higher prevalences and risk of transmission constitute a reservoir for sustaining the epidemic ${ }^{7}$.

A study conducted by Zewudie, Legesse, \&Kurkura (2002) in South-Western Ethiopia, reported that only $51 \%$ of barbers knew that HIV could be transmitted through barbing equipment, which is lower than the $63.6 \%$ found in the current study ${ }^{17}$. This difference could be due to the differences in the sample size, time of the study, prevalence of HIV/AIDS and geographical location between the two studies. However, the 63.6\% good knowledge regarding HIV/AIDS as reported in the current study can still be considered inadequate. Knowledge regarding HIV should be universal in order to effectively tackle the disease.

The significant association between educational /evel and level of knowledge found in the current storov is in accordance with a study conducted by N Nurbight and Sarfo (2015) in Obuasi, Ghana, which rdveated that the knowledge of the barbers increases with increasing educational level ${ }^{18}$.This is also similar to a descriptive cross-sectional study conducted by Adoba et al (2015), in Obuasi in the Ashanti Region, Ghana, where it was discovered that $52.2 \%$ of the barbers who had knowledge of viral infections had a post-secondary education $^{19}$. Education could decrease the transmission of viral-borne diseases as a result of barbers practicing good sterilization and disinfection procedures.

The current study found a significant association between barbers' knowledge and work experience. This is contrary to the observations by Wazir et al. $(2011)^{16}$ in Pakistan ${ }^{16}$, in which the level of knowledge among barbers about health hazards was poorly associated with their number of years in the profession. The reason for this discrepancy could be the demographic differences between the study area in Pakistan and Ho. Ho is largely a Christian area while Pakistan is largely a Muslim area.

Most of the participants in this current study did not know their HIV/AIDS status and would continue their profession if they were infected. This could pose a major risk to clients who patronize these barbers, whether or not they are symptomatic, and hence more aware- ness should be advocated to get the barbers to know their status and observe proper adherence to prevention practices in order to minimize the chances of HIV transmission.

The attitudes of the participants were generally favorable as a majority of the barbers had good attitudes regarding HIV/AIDS (58.7\%). This is in line with a previous study conducted in South-Western Ethiopia, which revealed that majority of barbers had favorable attitudes regarding HIV/AIDS $(67.8 \%)^{13}$. The favorable attitudes of the barbers in the current study may be due to their access to adequate information while learning the barbing profession, leading to knowledge on disease transmission caused by unsterile sharp equipment. However, it is worth noting that the $58.7 \%$ good attitude reported in this study is inadequate with respect to HIV/AIDS. To effectively tackle HIV/AIDS, there should be $\$ 00 \%$ good attitude towards the disease. Pooy attitudes towards HIV/AIDS could lead to stigma and diqcrimination against PLHIV. Just like with knowledge, the current study showed significant associations between attitude and work experience on one hand, and between attitude and level of education on the other. These positive associations could result from the significant relationship between knowledge and attitude asound in the current study, with barbers having good knowledge regarding HIV/AIDS more likely to have good attitudes towards HIV/AIDS.

Barbers in the current study manifested poor prevention practices regarding HIV/AIDS. This study demonstrated that barbers in the study area seemed to be practicing disinfection instead of sterilization. Micro-trauma induced while shaving causes release of blood and other bodily fluids, which can cause transmission of HIV and other virus such as HBV and HCV to barbers when they come in contact with these fluids. Also, contamination of the shaving instruments can pose a great risk to other clients. Despite this, few barbers in the current study were aware of the mode of transmission of HIV/ AIDS infections, and the risk posed by unsafe shaving practices. Most of the barbers were found to have UV lights in their barbers' shops but the bulbs were not frequently changed. This is in line with a study conducted in Obuasi, Ghana, which indicated that a UV radiation sterilizer cabinet was seen in all barbershops visited, but the majority of the sterilizers could only be described as storage cabinets or for display purposes as most of the sterilizer cabinets either used mercury bulbs instead of UV bulbs or did not have any light source in them ${ }^{14}$. 
These flawed professional practices could be attributed to lack of information about the sterilization process. Most barbers were seen placing their hair trimmers into the sterilizer cabinets when not in use or after use on a client. However, for those barbershops with heavy workloads the same set of hair trimmers could be used continuously for multiple clients without sterilization ${ }^{17}$.

Skin damage is the prerequisite for inoculation of the scalp with HIV; penetration of the skin with barbing instruments exposes the circulatory system to infection. Skin damage occurs during barbing as an accidental cut or abrasion resulting from blade-to skin contact. Bladeto-scalp contact often occurs during hair shaping, shaving and zero-hair cutting involving use of a detachable plastic comb ${ }^{17}$.

Studies carried out in Morocco, Ethiopia and Pakistan showed that the level of knowledge, awareness and practices of barbers about the concept of infectious risk associated with blood was generally very $\operatorname{low}^{18}$. This is similar to findings of the current study where a majority of study participants were aware of HIV/AIDS but lacked adequate sterilization practices and did not associate their practices with the risk of transmission. It is also in line with the study by Belbacha, Cherkaoue, Akrim, Dooley and El-Aouad (2012), which found that traditional barbers and their clients in Morocco are unfamiliar with proper practices and are mostly unaware of the transmission of blood borne pathogens through shaving tools ${ }^{19}$.

For these reasons, awareness campaigns are impeptive and should focus on both barbers and thegerier 1 population, especially those who are at risk du to their occupation. Also, for other viral diseases easily transmitted through the barbing process, such HBV and HCV, training and vaccination should be encouraged in order to curb the increasing incidence of these viral blood borne diseases ${ }^{20}$.

A possible reason for the poor HIV-prevention practices as reported in the current study may be the lack of strict control measures and monitoring by relevant bodies. Unlike in developed countries where activities of barbers are regulated through comprehensive training, licensing and monitoring programs, barbers in Ghana have not been given any noticeable attention to their profession and their activities ${ }^{10}$.

There was a significant association between location and level of practice, with more barbers in the rural are- as demonstrating good prevention practices than those in the urban areas. This is contrary to a study conducted in Nigeria to assess the potential risk of HIV transmission in barbing practices among professional barbers in Ibadan, Nigeria, which revealed that barbers in the high-class urban areas were more likely to practice appropriate equipment decontamination than those from the rural location ${ }^{20}$. The difference could be attributed to the fact that Ho municipality is comprised of more rural communities than the city of Ibadan in Nigeria.

Majority of the barbers with poor practices were found to be to aged 20-29 years. This is in line with a cross-sectional study conducted by Dongdem et al. (2013), to estimate the prevalence of $\mathrm{HIV} / \mathrm{HBV} / \mathrm{HCV}$ among blood donors at the Tamale Teaching Hospital in Ghana, which observed that the highest prevalence of $\mathrm{HIV} / \mathrm{HBV} / \mathrm{HCV}$ were among donors within the ages of $20-29^{21}$. This data supports findings from this study that barbers'practices could serve as a major route of transmission of HIV/AIDS. Barbers with more than 10 years' work experience were more likely to exhibit good prevention practices than those with less than 5 years' work experience.

\section{Limitations}

Most participants were reluctant to participate in the study because they thought that they were going to be sanctioned if it was found out that they were not licensed to practice. Those who also consented to participate were afraid that if their poor practices were reverted, the public health authorities would halt their operations. Participants could have answered the questions to their advantage since observation was not used as a tool to collect data because the study design was quantitative. Furthermore, the exclusion of road-side barbers could have introduced in a selection bias. However, against the above limitations, this study stands as the first describing the knowledge, attitudes and prevention practices regarding HIV/AIDS among barbers in Ho municipality, Ghana, which could be used for planning, programming and further research targeting this high risk group. Additionally, the cluster samplingincreased the representational character of the population of the study, despite the small sample size of 121 .

\section{Conclusion}

Barbers involved in the study had relatively good knowledge and attitudes regarding HIV/AIDS, though they could still be considered inadequate, and also exhibited poor prevention practices regarding HIV/AIDS. Knowledge was influenced by level of education, work 
experience and number of clients seen per day, while attitude was influenced by work experience, mode of training, level of education and knowledge. Practice was influenced by work experience and community of operation.

As a recommendation, we suggest that awareness campaigns in HIV prevention in Ho Municipality, Ghana, must include the barbers in order to bridge their knowledge gaps and change their practices. All associated factors identified must be incorporated into those campaigns to tailor them to the local setup.

\section{Conflict of interest}

None declared.

\section{References}

1. Global AIDS Update 2016 [Internet]. [cited 2018 Jun 8]. Available from: http://www.unaids.org/en/resources/documents/2016/Global-AIDS-update-2016

2. Patel R, Choudhary N, Bhat N, Reddy JJ, Rav N, Goyal N, et al. Knowledge, Attitudes and Practice of Baebers regarding $\mathrm{HIV} / \mathrm{HBV} / \mathrm{HCV}$ infections of Udaipur City, Rajasthan, India. Int J Oral Health Med Res. 2015;2(1):27-31.

3. S C, Khan K. Sharing razor blades in salons and risk of spreading HIV in Bangladesh. ,. In: The $3^{\text {rd }}$ IAS Con ference on HIV Pathogenesis and Treatnent. Bariala desh; 2004. p. 02. (Poster Exhibition).

4. Biadgelegn F, Belyhun Y, Anagaw B, Wo Geyohannes D, Moges F, Bekele A, et al. Potential risk of HIV transmission in barbering practice in Ethiopia: from public health and microbiological perspectives. BMC Public Health. 2012 Aug 29;12:707.

5. HUMPHRIES G. I determine the following code of practice under the Public Health Act 1997. Dated 12 January 2000. Public Health. 2000;15.

6. Khandait DW, Ambadekar NN, Vasudeo ND. Knowledge and practices about HIV transmission among barbers of Nagpur City. Indian J Med Sci. 1999 Apr;53(4):167-71.

7. Addo N, Yawson A, Addo AS, Dornoo BT, Seneadza HNA. A Review of National Programme Data on the HIV Epidemic in Ghana: 2005-2010. Posgraduate Med J Ghana. 2014;3:73-81 PubMed .

8. 2010 Population And Housing Census, Ghana | Statistical Data Sets | Environmental Social Science [Internet]. Scribd. [cited 2018 Jun 8]. Available from: https:/ / www.scribd.com/document/218586577/2010-Population-And-Housing-Census-Ghana

9. Cochran WC. Snedecor G W \& Cochran W G. Sta- tistical methods applied to experiments in agriculture and biology. 5th ed. Ames, Iowa: Iowa State University Press, 1956. :1.

10. Mutocheluh M, Kwarteng K. Knowledge and occupational hazards of barbers in the transmission of hepatitis B and C was low in Kumasi, Ghana. Pan Afr Med J. 2015;20:260.

11. Chanda S, Khan K. Sharing razor blades in salons and risk of spreading HIV in Bangladesh. In.

12. Gss GSS-, Ghs GHS-, International ICF. Ghana Demographic and Health Survey 2014. 2015 [cited 2018 Jun 8]; Available from: http://dhsprogram.com/ publications/publication-fr307-dhs-final-reports.cfm

13. Zewudie T, Legesse W, Kurkura G. Knowledge, attitudes and Practices among barbers in South-western Ethiopia. Afr Newslett on Occup Health and Safety. 2002;12(3):69-71.

14. Akumiah PO. Sarfo LA. Knowledge and Practice of Certified Barbers About Hepatitis B and C Transmission in Kumasi, Ghana. ARJ. 2015, 1(2): 27-35

15. Adoba P, Boadu SK, Agbodzakey H, Somuah D, Ephraim RKD, Odame EA. High prevalence of hepatitis B and poor knowledge on hepatitis B and C viral infections anong barbers: a cross-sectional study of the Obuasi numicipality, Ghana. BMC Public Health. 2015 Ocd 1 1, 15:1041.

16. Wazir MS, Mehmood S, Ahmed A, Jadoon HR. Awareness among barbers about health hazards associated with their profession. J Ayub Med Coll Abbottabad. 2008;20(2):35-8.

17. Arulogun OS, Adesoro MO. Potential risk of HIV transmission in barbering practice among professional barbers in Ibadan, Nigeria. Afr Health Sci. 2009 Mar;9(1):19-25.

18. Khuwaja AK, Qureshi R, Fatmi Z. Knowledge about hepatitis $\mathrm{B}$ and $\mathrm{C}$ among patients attending family medicine clinics in Karachi. East Mediterr Health J. 2002 Nov;8(6):787-93.

19. Belbacha I, Cherkaoui I, Akrim M, Dooley KE, El Aouad R. Seroprevalence of hepatitis B and C among barbers and their clients in the Rabat region of Morocco. East Mediterr Health J. 2011 Dec;17(12):911-9.

20. Arulogun OS, Adesoro MO. Potential risk of HIV transmission in barbering practice among professional barbers in Ibadan, Nigeria. Afr Health Sci. 2009 Mar;9(1):19-25.

21. Dongdem JT, Kampo S, Soyiri IN, Asebga PN, Ziem JB, Sagoe K. Prevalence of hepatitis B virus infection among blood donors at the Tamale Teaching Hospital, Ghana (2009). BMC Res Notes. 2012 Feb 22;5:115. 